\title{
Tratamiento quirúrgico de la incontinencia urinaria de esfuerzo mediante cabestrillo suburetral SPARC. Análisis de nuestra experiencia
}

\author{
Ponce Díaz-Reixa J, Barbagelata López A, Álvarez Castelo L, Romero Selas E, \\ Sánchez Rodríguez-Losada J, Fernández Rosado E, Montes Couceiro M, González Martín M.
}

\author{
Servicio de Urología. Hospital Juan Canalejo. A Coruña.
}

Actas Urol Esp. 2007;31(10):1129-1133

\section{RESUMEN}

TRATAMIENTO QUIRÚRGICO DE LA INCONTINENCIA URINARIA DE ESFUERZO MEDIANTE CABESTRILLO SUBURETRAL SPARC. ANALISIS DE NUESTRA EXPERIENCIA

Introducción: La incontinencia urinaria de esfuerzo (IUE) presenta una gran morbilidad y una elevada incidencia. Existen múltiples técnicas quirúrgicas descritas para el tratamiento de la misma, con diversos resultados. Los cabestrillos suburetrales se han establecido en los últimos años como un método simple y poco invasivo para el tratamiento de la incontinencia urinaria de esfuerzo.

Material y Método: Analizamos retrospectivamente nuestra serie de 86 pacientes diagnosticadas de IUE, intervenidas, entre Octubre de 2001 y Diciembre de 2005, mediante un cabestrillo suburetral suprapúbico (SPARC@).

Resultados: La media de edad es de 58,7 años (39-80). La estancia media hospitalaria es de 2,43 días (1-8) y la duración media de sonda de 1,52 días (1-10). En el 14,5\% de los casos, se realizó cirugía asociada, siendo la más frecuente, la colpoperineoplastia anterior (10,7 \%). Las complicaciones son poco frecuentes; perforación vesical (5,8\%), RAO antes del alta (7\%), ITU (15,3\%), RAO crónica (3,5\%). En el 3,5\% de las mujeres, se realizó lisis del cabestrillo, por retención crónica. El seguimiento medio es de 10,14 meses, encontrándose un 71,4\% de continencia total. Un $26,2 \%$ de las pacientes presentaron sintomas de hiperactividad de novo, con problemas de incontinencia en el 54,5\% de estas. (p<0,002; OR 5.0 (IC 95\% 1,75-14,28).

Conclusiones: El SPARC@ es un método sencillo, con un tiempo hospitalario corto y una rápida reincorporación a la vida social. Los resultados pueden ser valorados rápidamente y con una tasa elevada de éxito. La aparición de urgencia de novo es elevada y empeora los resultados funcionales. Es necesario un mayor seguimiento para valorar resultados funcionales a largo plazo.

Palabras clave: Incontinencia urinaria de esfuerzo. Cabestrillo suburetral.

\begin{abstract}
STRESS URINARY INCONTINENCE SURGICAL TREATMENT WITH SUBURETHRAL SUPRAPUBIC SPARC SLING

Introduction: Stress urinary incontinence (SUI) has a high incidence and important morbidity. Multiple surgical techniques have been described to treat it, with despair results. Suburethral slings have become, in recent years, a simpler and less invasive method to treat SUI surgically.

Material and method: The purpose of this paper is to review, retrospectively, 86 patients treated at our institution, from 10/01 to 12/05 of SUI, with a suprapubic suburethral sling (SPARC).

Results: Medium (range) age is 58.7 (39-80), hospital stay was $2.43 \mathrm{~d}$ (1-8) and catheter was removed $1.52 \mathrm{~d}$ (1-10). $14.5 \%$ of patients underwent other vaginal surgeries at same time, most frequently anterior colpoperineoplasty (10.7\%). Complications are not very frequent; bladder perforation (5.8\%), acute urinary retention $(7 \%)$, chronic urinary retention (3.5\%) and UTI (15.3\%). Sling release was performed in 3.5\% of women with chronic urinary retention. Total continence was found in $71.4 \%$ of patients with 10.1 months medium follow up (1-32). De novo urge symptoms were found in $26.2 \%$ of women, with urge incontinence in $54.5 \%$ of them. (p < 0,002; OR 5.0 (IC 95\% 1.75-14.28).

Conclusions: Suprapubic suburethral SPARC sling is a simple method, with few complications and fast social recovery. Outcome can be measured soon, with a high continence rate. De novo urge symptoms are high and they worsen functional results. It is necessary longer follow-up to evaluate long term outcomes.
\end{abstract}

Keywords: Stress urinary incontinence. Suburethral slings. 
$\mathrm{L}$ a incontinencia urinaria de esfuerzo (IUE) presenta una gran morbilidad y una elevada incidencia. Para ser continente, se precisa que la capacidad de cierre de la uretra, sea superior a la presión vesical. El cierre se mantiene por la acción adecuada de unas fuerzas pasivas y activas. En el primer grupo, incluimos la normalidad de estructuras anatómicas y el correcto soporte de la uretra durante los esfuerzos. Los elementos activos son una adecuada inervación uretro-vesical y la musculatura del esfinter y el suelo pélvico.

Se ha postulado diversas hipótesis para entender la IUE por fallo de los mecanismos de soporte. La teoría de Hodkingson ${ }^{1}$ por la insuficiente transmisión de los aumentos de presión abdominal a la uretra durante el esfuerzo. La teoría de DeLancey ${ }^{2}$, en la que el soporte vaginal uretral está debilitado durante los esfuerzos y la teoría de Petros $^{3}$ en que se entiende la IUE por una alteración en el soporte del tercio medio de la uretra. Esta última teoría es la base para el tratamiento quirúrgico de las bandas libres de tensión.

La técnica de colocación de los cabestrillos suburetrales, fue descrita inicialmente por Ulmsten en 1996 con la colocación de mallas de TVT $^{4}$. Es una intervención que presenta seguridad y efectividad en sus resultados para el tratamiento de la incontinencia urinaria de esfuerzo genuina, siendo mínimamente invasiva con buenos resultados a largo plazo ${ }^{5}$.

El cabestrillo SPARC(C) (American Medical Systems) es una malla de polipropileno que utiliza un abordaje suprapúbico para el tratamiento de la IUE secundaria a hipermobilidad uretral o una deficiencia esfinteriana intrínseca. Utiliza dos agujas finas y curvas que permiten el paso de la malla. Ésta soporta la uretra durante las elevadas presiones abdominales y su porosidad permite una buena integración tisular y cicatrización.

\section{MATERIAL Y MÉTODOS}

Se realiza un estudio retrospectivo, de los resultados quirúrgicos, en el tratamiento de la IUE femenina. Analizamos 86 pacientes diagnosticadas de IUE genuina e intervenidas, entre Octubre de 2001 hasta Diciembre de 2005. En todas las pacientes, se utilizó el dispositivo suburetral suprapúbico SPARC.
Las pacientes son valoradas en la unidad de Urodinámica de nuestro centro, realizándose anamnesis y exploración física general y ginecológica. Se descarta inestabilidad del detrusor, mediante exploración urodinámica. La intervención es realizada bajo anestesia regional en todos los casos. Se pauta profilaxis antibiótica con Ciprofloxacino y profilaxis tromboembólica (HBPM) en todos los casos. La técnica se realiza con el paso de agujas de forma descendente, comprobando posteriormente, mediante cistoscopia, la indemnidad de la pared vesical. Se asciende el cabestrillo suburetral, dejándolo libre de tensión, en el tercio medio de la uretra. Dejamos sonda vesical y taponamiento vaginal durante 24 horas.

Las pacientes son seguidas de forma ambulatoria al mes, 3, 6 y 12 meses y luego anualmente. Los resultados son valorados clínicamente, no se utiliza test subjetivo ni objetivo. Consideramos pacientes continentes aquellas que no refieren pérdida con los esfuerzos. Las complicaciones precoces son las consideradas ocurridas en el postoperatorio inmediato, antes del alta hospitalaria. Las complicaciones tardías son evaluadas en la consulta externa.

El análisis estadístico, se realiza con el programa informático SPSS 12.0 de Windows. Para variables cuantitativas, usamos la t de Student y para variables cualitativas, la Chi Cuadrado. Se consideran diferencias significativas, los valores de $\mathrm{p}<0,05$.

La media de edad es de 58,71 + 9,63 años (3980). La duración media de la sonda vesical postoperatoria es de 1,52 \pm 1,51 dias (1-10) y la media de estancia hospitalaria es de $2,43 \pm 1,53$ dias ( 1 8). El tiempo medio de seguimiento es de 10,14 \pm 8,46 meses (1-32) (Tabla 1).

\section{RESULTADOS}

En el 14,5\% de las intervenciones, se realizó cirugía asociada. La corrección del prolapso anterior, mediante colpoperineoplastia anterior $(10,7 \%)$

Tabla 1. Descriptivo de los 86 pacientes

\begin{tabular}{lccccc}
\hline & Media \pm DE & Media \pm DE & Mediana & Min & Máx. \\
\hline Edad & 58,71 & 9,63 & 59 & 39 & 80 \\
Días de sonda & 1,52 & 1,51 & 1 & 1 & 10 \\
Días de estancia & 2,43 & 1,53 & 2 & 1 & 8 \\
Seguimiento & 10,14 & 8,46 & 8 & 1 & 32 \\
\hline
\end{tabular}


fue la más frecuente; prolapso posterior $(3,5 \%)$, prolapso anterior y posterior $(3,5 \%)$ e histerectomía vaginal $(1,2 \%)$.

Las complicaciones intrahopitalarias $(17,4 \%)$, se muestran en las Tabla 2. La RAO al retirar la sonda, se presentó en el 7\% de las pacientes, tratadas inicialmente de forma conservadora, con sonda vesical o cateterismo intermitente. En el $3,5 \%$ de las pacientes, se comprobó, en el seguimiento posterior, la persistencia de retención urinaria, con residuos altos, conllevando estas pacientes, la lisis del SPARC. Con anestesia regional, seccionamos la malla a ambos lados de la uretra. Estas tres pacientes recuperaron un vaciamiento vesical adecuado, sin pérdidas y con imperiosidad en una de ellas.

Tabla 2. Complicaciones precoces (Intrahospitalarias)

\begin{tabular}{lcc}
\hline RAO & 6 & $7,0 \%$ \\
Fiebre & 1 & $1,2 \%$ \\
Sangrado Vaginal & 2 & $2,3 \%$ \\
Hematuria & 1 & $1,2 \%$ \\
Perforación vesical & 5 & $5,8 \%$ \\
Total & $\mathbf{1 5}$ & $\mathbf{1 7 , 4} \%$ \\
\hline
\end{tabular}

En el 5,8\%, se observo perforación vesical intraoperatoria, tratada mediante recolocación de las agujas en sentido descendente, con posterior control cistoscópico. En todas se pudo colocar el cabestrillo sin problemas. Dos pacientes $(2,3 \%)$ presentaron sangrado vaginal moderado y una paciente, con perforación vesical corregida, hematuria autolimitada leve $(1,2 \%)$. Ninguna de estas pacientes precisó transfusión sanguínea. Una paciente presentó fiebre en el primer día postoperatorio, que se trató empíricamente, sin incidencias.

Las complicaciones extrahospitalarias (Tabla 3) $(23,5 \%)$, tienen su mayor exponente en la ITU $(15,3 \%)$, tratada con antibioterapia empírica y con urocultivo de control. Cabe destacar también, que en una paciente $(1,3 \%)$, se observó extrusión de los bordes de la malla por la incisión vaginal, realizándose exéresis de la malla. cualitativas
Tabla 3. Complicaciones Tardías (Extrahospitalarias)

\begin{tabular}{lcc}
\hline ITU & 13 & $15,3 \%$ \\
RAO & 3 & $3,5 \%$ \\
Lisis SPARC & 3 & $3,5 \%$ \\
Extrusión bordes vaginal & 1 & $1,2 \%$ \\
Total & $\mathbf{2 0}$ & $\mathbf{2 3 , 5} \%$ \\
\hline
\end{tabular}

Con un seguimiento medio de 10,1 meses (1-32), la continencia total se observo en el $71,4 \%$ de las pacientes. No se realizó test objetivo de la misma, sino la percepción clínica de cada paciente. En un $26,2 \%$ de las mujeres, hemos observado hiperactividad de novo, produciendo incontinencia en el 54,5\%; con diferencias significativas, frente a las mujeres sin hiperactividad de novo. El riesgo de presentar incontinencia es cinco veces superior, cuando aparece clínica de hiperactividad de novo (p < 0,002; OR 5,0 (IC 95\% 1,75-14,28).

Realizamos un análisis estadístico, en el que se observa que las variables descriptivas no demuestran diferencias estadísticas frente a la aparición de incontinencia. Las pacientes continentes, tienen un tiempo de seguimiento menor, que las incontinentes $(8,9 \mathrm{~m}$ frente a $13,1 \mathrm{~m}$, $\mathrm{p}=0,03$ ). Esto es debido a un mayor seguimiento de las pacientes incontinentes y altas precoces cuando la paciente ya se encuentra totalmente continente (Tabla 4).

Realizamos también un análisis de factores que pudieran predecir la aparición de hiperactividad de novo, valorando variables cuantitativas (edad, días de sonda, días de estancia) y variables cualitativas (complicaciones y cirugías asociadas). Vemos que en el primer grupo, no se encuentran diferencias significativas. En el segundo grupo, analizamos

Tabla 4. Análisis estadístico de la continencia, según variables cuantitativas y

\begin{tabular}{|c|c|c|c|c|c|}
\hline & \multicolumn{2}{|c|}{ Continencia } & \multicolumn{2}{|c|}{ Incontinencia } & \multirow[b]{2}{*}{ Valor $\mathbf{p}$} \\
\hline & Media $\pm \mathrm{DE}$ & Media $\pm \mathrm{DE}$ & Media $\pm \mathrm{DE}$ & Media $\pm \mathrm{DE}$ & \\
\hline Edad & 59,55 & 9,9 & 56,46 & 9,1 & ns \\
\hline Días de sonda & 1,40 & 1,1 & 1,63 & 1,9 & ns \\
\hline Días de estancia & 2,30 & 1,4 & 2,54 & 1,5 & ns \\
\hline Seguimiento & 8,93 & 7,9 & 13,17 & 9,1 & 0,037 \\
\hline \multicolumn{2}{|c|}{ Hiperactividad de Novo } & $\%$ & \multicolumn{2}{|r|}{$\%$} & $\mathbf{p}$ \\
\hline \multicolumn{2}{|l|}{$\mathrm{Si}$} & $45,5 \%$ & \multicolumn{2}{|r|}{$54,5 \%$} & $<0,002$ \\
\hline \multicolumn{2}{|l|}{ No } & $80,6 \%$ & \multicolumn{2}{|r|}{$19,4 \%$} & \\
\hline
\end{tabular}


la aparición de complicaciones precoces $(17,4 \%)$ o tardías $(23,5 \%)$, no observando diferencias significativas. Asimismo, la conjunción de cirugias en el mismo acto quirúrgico, tampoco observa diferencias estadísticas, para la aparición de hiperactividad de novo (Tabla 5).

\section{DISCUSIÓN}

Las mallas tipo sling de polipropileno son frecuentes en el tratamiento quirúrgico de la IUE. Desde la introducción de la TVT en 1996, la técnica ha ido evolucionando. El dispositivo suburetral SPARC presenta diversas diferencias y ventajas frente a la TVT ${ }^{16}$. El calibre de las agujas y su introducción suprapúbica, permite al cirujano seguirlas en todo su recorrido cerca del pubis y guiarse con el dedo en la incisión vaginal.

Existes múltiples series nacionales publicadas con el TVT, con resultados de continencia entre 65-96\% ${ }^{6-13}$ La serie publicada por Borrel et al., analiza la colocación de 17 cabestrillos SPARC, con un $94 \%$ de curación completa, 5,8\% de perforación vesical y $11,7 \%$ de hiperactividad de novo ${ }^{14}$. Andonian et al. realiza un estudio prospectivo y randomizado, de 84 pacientes, para comparar el dispositivo suprapúbico SPARC y el TVT. Ambos dispositivos no presentan diferencias

Tabla 5. Análisis estadístico de la hiperactividad, según variables

\begin{tabular}{|c|c|c|c|c|c|}
\hline & \multicolumn{5}{|c|}{ Hiperactividad de Novo } \\
\hline & \multicolumn{2}{|c|}{ No } & \multicolumn{2}{|c|}{$\mathbf{S i}$} & \multirow[b]{2}{*}{ Valor $\mathbf{p}$} \\
\hline & Media $\pm \mathrm{DE}$ & Media $\pm \mathrm{DE}$ & Media \pm DE & Media \pm DE & \\
\hline Edad & 58,74 & 9,7 & 58,45 & 9,9 & ns \\
\hline Días de sonda & 1,55 & 1,6 & 1,23 & 0,5 & $\mathrm{~ns}$ \\
\hline Días de estancia & 2,37 & 1,7 & 2,36 & 1,3 & ns \\
\hline \multicolumn{2}{|l|}{ Complicaciones } & \multicolumn{3}{|c|}{ Hiperactividad de Novo } & Valor $\mathbf{p}$ \\
\hline \multicolumn{2}{|l|}{ Precoces } & \multicolumn{2}{|l|}{ No } & Si & \\
\hline \multicolumn{2}{|l|}{ No } & \multicolumn{2}{|l|}{$72,5 \%$} & $27,5 \%$ & ns \\
\hline \multicolumn{2}{|l|}{$\mathrm{Si}$} & \multicolumn{2}{|l|}{$80 \%$} & $20 \%$ & \\
\hline \multicolumn{2}{|l|}{ Tardias } & \multicolumn{2}{|l|}{ No } & $\mathbf{S i}$ & Valor $\mathbf{p}$ \\
\hline \multicolumn{2}{|l|}{ No } & \multicolumn{2}{|l|}{$76,6 \%$} & $23,4 \%$ & ns \\
\hline \multicolumn{2}{|l|}{$\mathrm{Si}$} & \multicolumn{2}{|l|}{$65 \%$} & $35 \%$ & \\
\hline \multirow{2}{*}{\multicolumn{2}{|c|}{ Cirugias Asociadas }} & \multicolumn{3}{|c|}{ Hiperactividad de Novo } & Valor $\mathbf{p}$ \\
\hline & & \multicolumn{2}{|l|}{ No } & $\mathbf{S i}$ & \\
\hline \multicolumn{2}{|l|}{ No } & \multicolumn{2}{|l|}{$71,8 \%$} & $28,2 \%$ & ns \\
\hline \multicolumn{2}{|l|}{$\mathrm{Si}$} & $84,6 \%$ & & $15,4 \%$ & \\
\hline
\end{tabular}

significativas en cuanto a complicaciones perioperatorias, estancia hospitalaria, perforación vesical, sangrado quirúrgico y retención urinaria. Con un seguimiento mínimo de 1 año, no hay tampoco diferencias en las tasas de curación ${ }^{15}$.

En una amplia serie retrospectiva de 445 pacientes tratadas con el cabestrillo SPARC, el $22 \%$ asoció cirugía de prolapso o uterina. La tasa de curación clínica es del $83 \%$. La perforación vesical ocurrió en el 6,7\% de ellas. Un 6,6\% presento retención crónica, requiriendo lisis del SPARC en el 4,3\%. Estos datos concuerdan con los presentados en nuestra serie. La hiperactividad de novo, se presentó solo en el 6,1\%, frente al $26,2 \%$ de nuestra serie. En el $4,3 \%$ se precisó seccionar el sling por retención crónica ${ }^{16}$.

En un estudio multicéntrico realizado en hospitales franceses, y publicado por Deval et al., se evalúa la eficacia y seguridad del cabestrillo SPARC. En el periodo de un año, se intervienen 104 pacientes. La media de seguimiento es similar a la nuestra (11,9 meses). La tasa de complicaciones es del $44,2 \%$, incluyendo un $10,5 \%$ de perforaciones vesicales, moderamente superior a nuestra serie $(5,8 \%)$. La tasa de curación difiere estadísticamente entre valoraciones objetivas y subjetivas $(90,4$ frente a $72 \%, \mathrm{p}<0,05)$. El 11,5\% de las pacientes presentó urgencia de novo, motivando incontinencia en el $42 \%$ de ellas. En nuestro caso, la urgencia de novo, es superior $(26 \%)$, y ligeramente superior la incontinencia en estas pacientes $(54,5 \%)^{17}$.

Wang et al. comparan de forma de manera prospectiva el dispositivo transobturador (MONARC) y suprapúbico (SPARC), en 60 pacientes. No encuentran diferencias estadísticas, pero si observan una clara diferencia en cuanto a perforación vaginal $(12,9 \%$ vs $0 \%)$ y dolor por presión a nivel uretral $(12,9 \%$ vs $0 \%)$ del dispositivo transobturador, frente al suprapúbico $^{18}$.

La tasa de continencia que hemos observado en nuestras pacientes $(71,4 \%)$, no son equiparables a 
otras series publicadas sobre SPARC $^{14-17}$. Las tasas de hiperactividad de novo de nuestra serie, son considerablemente superiores a las publicadas por otros autores ${ }^{17}$. En nuestro caso, la aparición de sintomas de urgencia de novo, presenta tasas de incontinencia elevadas $(54,5 \%)$, siendo estadísticamente significativas y con un riesgo 5 veces superior, frente a las pacientes sin urgencia de novo.

Este trabajo retrospectivo, tiene varias limitaciones en la interpretación de sus resultados. La primera es un seguimiento corto $(10,1 \mathrm{me}$ ses) y la falta de un seguimiento a largo plazo, que nos permita valorar su efectividad. La segunda es la falta de un test objetivo o subjetivo que permita valorar los resultados funcionales de la técnica. Es necesaria una publicación posterior en la que podamos comunicar resultados a largo plazo mediante un test objetivo o subjetivo.

\section{CONCLUSIONES}

El cabestrillo suburetral suprapúbico SPARC es una técnica quirúrgica que consigue elevadas tasas de continencia, en un procedimiento poco invasivo y que presenta bajas complicaciones. La aparición de urgencia de novo es elevada y empeora los resultados funcionales. Es necesario un mayor seguimiento para valorar resultados a largo plazo.

\section{REFERENCIAS}

1. Hodgkinson CP, Doub HP. Roentgen study of urethrovesical relationships in female urinary stress incontinence. Radiology. 1953;61(3):335-345.

2. DeLancey JO. Structural support of the urethra as it relates to stress urinary incontinence: the hammock hypothesis. Am J Obstet Gynecol. 1994 Jun;170(6):1713-1720; discussion 1720-1723.

3. Petros PE, Ulmsten UI. An integral theory of female urinary incontinence. Experimental and clinical considerations. Acta Obstet Gynecol Scand Suppl. 1990;153:7-31.

4. Ulmsten U, Petros P. Intravaginal slingplasty (IVS): an ambulatory surgical procedure for treatment of female urinary incontinence. Scand J Urol Nephrol. 1995;29(1):75-82

5. Nilsson CG, Kuuva N, Falconer C, Rezapour M, Ulmsten U. Long-term results of the tension-free vaginal tape (TVT) procedure for surgical treatment of female stress urinary incontinence. Int Urogynecol J Pelvic Floor Dysfunct. 2001;12 Suppl 2:S5-8.

6. Hualde Alfaro A, Jiménez Calvo J, Sarmiento Gómez C, Pinos Paul M, de Pablo Cárdenas A, Ripa Saldias L, et al. TVT our experience five years an six months later. Actas Urol Esp. 2006;30(2):181-185.
7. Quicios Dorado C, Fernández Fernández E, Gómez García I, Perales Cabanas L, Arias Fúnez F, Escudero Barrilero A. Treatment of female stress urinary incontinence with TVT system (tension-free vaginal tape): complications in our first 100 cases. Actas Urol Esp. 2005;29(8):750-756.

8. Borrell Palanca A, Chicote Pérez F, Beltrán Meseguer JF, Queipo Zaragozá JA, Esteve Claramunt J, Pastor Sempere F. Comparison of diferents suburethral slings for the treatment of stress urinary incontinence. Actas Urol Esp. 2005; 29(8):757-763.

9. Sanz Pérez G, Rodríguez-Rubio Cortadellas FI, Garrido Insua S, Concepción Masip T, Robles García JE, Gómez Velázquez M, et al. A Spanish multicenter trial of TVT for female urinary incontinence. Actas Urol Esp. 2005;29(7): 632-640.

10. Cañís Sánchez D, Bielsa Galí O, Cortadellas Angel R, Arango Toro O, Placer Santos J, Gelabert i Mas A. Results and complications of TVT procedure in the surgical treatment of female stress incontinence. Actas Urol Esp. 2005; 29(3):287-291.

11. Jiménez Calvo J, Hualde Alfaro A, Santiago González de Garibay A, Pinós Paul M, Jiménez Aristu J, Montesino Semper M, et al. TVT: three years of experience. Actas Urol Esp. 2004;28(1):13-20.

12. Briones Mardones G, Jiménez Cidre M, Fernández Fernández E, Perales Cabanas L, Pozo Mengual B, Sanz Migueláñez JL, et al. Treatment of female stress urinary incontinence with the TVT (tension-free vaginal tape) system: our experience. Actas Urol Esp. 2001;25(6):423-429.

13. Navalón Verdejo P, Zaragozá Fernández C, Ordoño Domínguez F, Sánchez Ballester F, Juan Escudero J, Fabuel Deltoro $\mathrm{M}$, et al. Outpatient surgical treatment of female stress urinary incontinence. Arch Esp Urol. 2005; 58(9):915-922.

14. Borrell Palanca A, Chicote Pérez F, Beltrán Meseguer JF, Queipo Zaragozá JA, Esteve Claramunt J, Pastor Sempere F. Comparison of diferents suburethral slings for the treatment of stress urinary incontinence. Actas Urol Esp. 2005;29(8):757-763.

15. Andonian S, Chen T, St-Denis B, Corcos J. Randomized clinical trial comparing suprapubic arch sling (SPARC) and tension-free vaginal tape (TVT): one-year results. Eur Urol. 2005;47(4):537-541.

16. Hodroff MA, Sutherland SE, Kesha JB, Siegel SW. Treatment of stress incontinence with the SPARC sling: intraoperative and early complications of 445 patients. Urology. 2005;66(4):760-762.

17. Deval B, Levardon M, Samain E, Rafii A, Cortesse A, Amarenco G, et al. A French multicenter clinical trial of SPARC for stress urinary incontinence. Eur Urol. 2003;44 (2):254-258.

18. Wang AC, Lin YH, Tseng LH, Chih SY, Lee CJ. Prospective randomized comparison of transobturator suburethral sling (Monarc) vs suprapubic arc (Sparc) sling procedures for female urodynamic stress incontinence. Int Urogynecol J Pelvic Floor Dysfunct. 2006;17(5):439-443.

Correspondencia autor: Dr. J. Ponce Díaz-Reixa Servicio de Urología. Hospital Juan Canalejo Xubias de Arriba, 84 - 15006 La Coruña. Tel.: 981178000 E-mail autor: ponce@canalejo.org Información artículo: Original - Incontinencia urinaria femenina Trabajo recibido: julio 2006

Trabajo aceptado: octubre 2006 